\title{
A biologia do câncer de mama e testes moleculares de prognóstico
}

\author{
Lucas Delmonico, ${ }^{1 *}$ Gilda Alves, ${ }^{1}$ Luiz F. P. do Amaral ${ }^{2}$
}

\section{Resumo}

A detecção precoce do câncer de mama aumenta consideravelmente as chances de cura, porém a identificação de lesões iniciais ainda é um desafio. Os casos mais comuns de diagnóstico são lesões palpáveis e de estádio avançado. Dessa forma, a busca por marcadores e testes moleculares tumorais vem sendo amplamente explorada na tentativa de minimizar tratamentos agressivos e contribuir para a detecção precoce do câncer de mama. A dificuldade na clínica ainda é justamente determinar com segurança os marcadores moleculares que seriam capazes de diferenciar lesões benignas autênticas das pré-malignas e malignas. Com isso, os testes moleculares têm permitido responder perguntas sobre prognóstico tumoral, recidivas e outras variáveis do fenótipo tumoral. Entre estes testes, o Oncotype DX ${ }^{\mathrm{TM}}$, Mammaprint e Prosigna têm possibilitado que a quimioterapia se torne mais individualizada, precisa e, quando comprovada a sua não necessidade, extinguido situações desconfortáveis para as pacientes, além do melhor custo e economia para o sistema de saúde.

Descritores: Neoplasias da mama; Diagnóstico precoce; Biologia molecular.

\section{Abstract \\ The biology of breast cancer and molecular prog- nostic tests}

Early detection of breast cancer greatly increases the chances of cure, but the identification of early lesions is still a challenge. The most common cases of diagnosis are palpable and advanced stage lesions. Thus, the search for tumor molecular markers and tests has been widely explored in an attempt to minimize aggressive treatments and contribute to early detection of breast cancer. The main challenge still lies in determining with the necessary degree of confidence which molecular markers would allow for a distinction between authentic benign, pre-malignant, and malignant. Molecular tests have made it possible to answer questions about tumor prognosis, relapse, and other variables of tumor phenotype. Among these tests, Oncotype DX ${ }^{\mathrm{TM}}$, MammaPrint, and Prosigna have enabled a more individualized and precise chemotherapy, as well as, when it proves to be unnecessary, avoided uncomfortable situations for patients. They've also allowed for the increased adoption of cost-effective treatments in the healthcare system.

Keywords: Breast neoplasms; Early diagnosis; Molecular biology.
1. Laboratório de Marcadores Circulantes. Faculdade de Ciências Médicas. Universidade do Estado do Rio de Janeiro. Rio de Janeiro, RJ, Brasil.

2. Faculdade de Ciências Médicas. Universidade do Estado do Rio de Janeiro. Rio de Janeiro, RJ, Brasil.

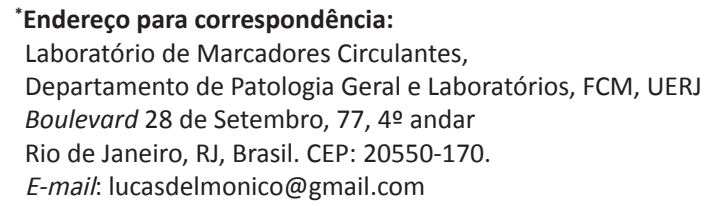

\section{Resumen}

\section{La biología de cáncer de mama y pruebas de pro-} nóstico moleculares

La detección temprana del cáncer de mama aumenta en gran medida las posibilidades de curación, pero la identificación de lesiones tempranas sigue siendo un desafío. Los casos más comunes de diagnóstico son en lesiones de etapa tangibles y avanzadas. Por lo tanto, la búsqueda de marcadores tumorales moleculares y pruebas ha sido ampliamente explorada en un intento por minimizar tratamientos agresivos y contribuir con la detección temprana del cáncer de mama. La dificultad en la práctica todavía es de determinar con precisión los marcadores moleculares con la confianza de que sean capaces de distinguir tumores auténticos benignos, premalignos y malignos. Por lo tanto, las pruebas moleculares han permitido responder a preguntas sobre el pronóstico del tumor, la recaída y otras variables del fenotipo tumoral. Entre estas pruebas, el Oncotype $\mathrm{DX}^{\mathrm{TM}}$, MammaPrint y Prosigna han permitido que la quimioterapia se vuelva más individualizada, y cuando sea demostrado necesaria o innecesaria, han disminuido situaciones incómodas para los pacientes, pero lo mejor es el ahorro de costos para el sistema de salud.

Palabras clave: Neoplasias de la mama; Diagnóstico precoz; Biología molecular. 


\section{Artigo de revisão}

\section{Visão geral do câncer de mama}

O câncer de mama é uma doença heterogênea, com apresentação e acompanhamento clínico bastante complexo. Corresponde a $29 \%$ de todos os tipos de tumores e é o segundo maior responsável pelas taxas de mortalidade globais relacionadas à carcinogênese. ${ }^{1}$ Somente no Brasil são esperados para o ano de 2015 57/100.000 casos. $^{2}$ Em consequência, patologistas e clínicos têm elaborado ferramentas na tentativa de padronização e adoção de tratamentos mais adequados, seguindo os indicadores preditivos de evolução, prognóstico e técnicas moleculares de diagnóstico do câncer.

Histologicamente, a mama é considerada uma glândula sudorípara constituída por ramificações de ductos e lóbulos. Apresenta compostos acinares, formados por células epiteliais e mioepiteliais envoltos por um estroma de tecido adiposo e tecido fibroso. Dentre o parênquima mamário é possível encontrar linfonodos intraductais e suas respectivas ramificações para as axilas. Pelas características morfológicas e unidades funcionais da mama, as lesões são originadas principalmente dos ductos e lóbulos.

Uma vez desenvolvidas, as lesões mamárias são classificadas como benignas (não neoplásicas e neoplásicas) e malignas. ${ }^{3}$ Engloba uma extensão de subtipos histológicos e são classificadas segundo o grau de diferenciação, proliferação e fenótipo celular. ${ }^{3}$ Em geral, essa classificação é adotada pela Organização Mundial da Saúde (OMS), ${ }^{4}$ e o grau nuclear por Elston e Ellis. ${ }^{5}$ Mais além, a positividade e negatividade dos receptores hormonais expressos no epitélio mamário (estrógeno, progesterona e human epidermal growth factor receptor 2 - HER2) irão determinar a conduta terapêutica do câncer de mama. Esta avaliação de expressão é realizada pela técnica de imunoistoquímica, que consiste na marcação do tecido mamário por anticorpos específicos.

\section{Diagnóstico por imagem}

Independentemente do avanço do diagnóstico molecular, o rastreamento e detecção precoce do câncer de mama é feito por imagem. A aplicação das técnicas moleculares ainda é complementar, justamente por não haver testes validados para rastreamento inicial. No cenário brasileiro, a idade de diagnóstico do câncer de mama tem diminuído ao decorrer dos anos, devido principalmente ao rastreamento da lesão pela mamografia e o desenvolvimento de programas assistenciais voltados à saúde da mulher. ${ }^{6}$ Infelizmente, o que é preconizado pelo Ministério da Saúde (MS) é o rastrea- mento anual por mamografia somente a partir dos 50 aos 69 anos para mulheres, ${ }^{2}$ o que contraria a idade ideal de início de rastreamento inicial do câncer de mama. ${ }^{6}$

Para o diagnóstico do processo carcinogênico mamário há três exames propedêuticos de imagens a serem realizados: mamografia (MA), ultrassonografia (US) e ressonância magnética (RM). Além destes, incluem os exames de alta tecnologia que incluem, por sua vez, imagens em 3D e mapeamento de alta resolução.

A MA é o único método atualmente considerado referência e adequado para rastreamento de mulheres assintomáticas. A sensibilidade da técnica varia entre 40-73\% e com uma especificidade de 35-98\% dependente da qualidade do equipamento utilizado e da densidade da mama. ${ }^{7,8}$ Além disso, já tem sido relatado um número considerável de casos com erros e divergências nos resultados da $\mathrm{MA}$, devido principalmente à inexperiência dos radiologistas. ${ }^{9}$

O exame mamográfico é realizado a partir de aparelhos de baixa intensidade de emissão de raios-x. A mama é comprimida sob um receptor de imagem (podendo ser de filme ou digital) e uma base transparente de compressão da mama. Os raios-x não absorvidos marcam o filme ou são revelados pelo detector digital produzindo uma fotografia similar a um negativo. ${ }^{10}$

A US tornou-se o exame mais sensível para o diagnóstico das doenças mamárias, com sensibilidade de 92\% e especificidade variando entre $90-96 \% .^{11} \mathrm{~A}$ técnica baseia-se nos transdutores de ondas com frequência média que varia de 5 para $15 \mathrm{MHz}$. Permite uma visualização em profundidade do tecido analisado e sua resolução garante a aplicação da técnica em mamas densas e em estágio de lactação. A emissão das ondas sonoras em diferentes ângulos recria a imagem com precisão, possibilitando detectar anormalidades na mama avaliada. ${ }^{12}$ Tornou-se uma ferramenta valiosa e complementar à mamografia na rotina, em especial para a detecção de carcinomas não palpáveis e ocultos clinicamente. Contudo, o seu uso élimitado na clínica, além de ser operador-dependente.

A ressonância nuclear magnética (RNM) atualmente é uma técnica utilizada para o acompanhamento do estadiamento local e vigilância da lesão mamária. Possui sensibilidade superior, ou mesmo equiparada à US, e especificidade inferior aos demais exames com $88 \% .^{13}$ É considerado um exame com necessidade de aprimoramentos técnicos para que seja mais amplamente utilizado. ${ }^{11}$

A técnica consiste na secção do tecido analisado em quadros de imagens com a recriação do movimento 
dos prótons de acordo com o relaxamento do tecido. O uso da RM no diagnóstico do câncer de mama ainda é limitado, especialmente em pacientes jovens com mamas densas e genes BRCA1 ou BRCA2 mutados, ou mesmo nos casos em que os genes não foram testados, porém de risco primário aumentado. ${ }^{14}$

Em aspectos complementares, o valor de diagnóstico da RNM é usado principalmente para:

a) detecção multifocal, multicêntrica e de patologia contralateral, ou quando a detecção por MA e US não foi suficiente para reconhecer a anormalidade mamária;

b) detectar a possibilidade de invasão tumoral em carcinoma ductal in situ;

c) avaliar a resposta à quimioterapia neoadjuvante;

d) detectar massas tumorais ocultas na mama, principalmente quando presentes inicialmente nos linfonodos axiliares;

e) detecção de lesões impalpáveis em mamas densas;

f) avaliar dinamicamente as curvas de washout das lesões mamárias. ${ }^{15}$

Considerando a capacidade de detecção de lesões ocultas na mama, foi demonstrado que a RM foi capaz de revelar 96\% das patologias mamárias multifocais/ multicêntricas, enquanto a MA e a US, comparadamente, foram capazes de detecção de somente $28,6 \%$ e $26,5 \%$, respectivamente. ${ }^{16}$

\section{Receptores hormonais}

Os estrogênios em geral são produzidos nos ovários e na placenta e, em menores quantidades, no tecido adiposo, testículos, vasos sanguíneos, mama e outros. No total, existem três compostos: o estradiol, o estrona e o estriol, sendo o estradiol o mais potente e o de maior produção nos ovários. ${ }^{17} \mathrm{O}$ receptor de estrogênio (RE) é dividido em dois subtipos - RE- $\alpha$ e RE- $\beta$-, sendo que ambos compartilham de estruturas funcionais similares. Atua como agente para crescimento normal e desenvolvimento do tecido mamário, mas os altos níveis deste composto elevam o risco do desenvolvimento para o câncer de mama. ${ }^{18}$ Isto se explica por três motivos:

a) o receptor RE- $\alpha$ se transloca para o núcleo promovendo o controle da expressão gênica;

b) produtos oxidativos gerados a partir do metabolismo do estrogênio promovem quebras e danos ao DNA;

c) a exposição excessiva do estrogênio leva a um crescimento desregulado das células expostas ao composto. $^{18}$

Já os receptores de progesterona (RP) se assemelham bastante aos de estrogênio, possuindo duas isoformas - $\mathrm{O}$ $\mathrm{RP}-\alpha$ e RP- $\beta$. Ambos possuem regiões estruturais equiparadas e com sítio de ligação ao DNA. Sob influência do hormônio progesterona, a porção de ligação se transloca no sentido nuclear, promovendo a regulação da expressão de genes-alvos. ${ }^{19}$

Em geral, os receptores de estrógeno e progesterona são fatores preditivos de resposta à hormonioterapia. A positividade dos receptores depende da idade e período menstrual da mulher, sendo que em média, 30\% apresentam RE positivo. Esta porcentagem é reduzida em $10-20 \%$ nas mulheres em período de pré-menopausa e se eleva a 50-60\% nas mulheres em período de pósmenopausa. $\mathrm{O}$ valor prognóstico dos receptores de estrogênio é diversificado entre os estudos, porém é relatado que pacientes com RE positivo apresentam tempo de sobrevida livre da doença maior que pacientes RE negativo. Desta forma, tem-se sugerido que o RE esteja mais associado ao crescimento celular do tumor, do que propriamente um marcador de potencial metastático..$^{20-23}$

Várias modalidades de hormonoterapia já foram utilizadas no tratamento do câncer de mama, utilizando em especial antiprogestágenos. Atualmente, o usual é utilizar de moduladores seletivos para receptores de estrógeno, como tamoxifeno, inibidores de aromatase e análogos de hormônio da gonatropina, justamente pela seletividade e participação protagonista no câncer. ${ }^{19}$ É regulamentado pelo College American of Pathologists (CAP) que se faça a pesquisa de expressão dos receptores hormonais em todos os tipos de tumores mamários, inclusive em carcinomas ductais in situ (CDIS). ${ }^{24} \mathrm{~A}$ hormoniterapia é indicada para qualquer paciente que apresente expressão dos receptores esteroídicos de forma adjuvante, ou mesmo de forma paliativa em tumores em processo metastático.

Os estudos que detalham a identificação do gene codificador de HER2 tiveram início em $1985 .{ }^{25}$ Com características similares aos genes da família de crescimento epidérmico, o gene foi isolado a partir de células tumorais do sistema nervoso central e, então, denominado NEU. Em 1988, Van de Vijver e colaboradores ${ }^{26}$ deram os primeiros passos na identificação da influência do gene no câncer de mama. No subtipo comedo, o estudo observou uma forte marcação da proteína HER2 no tecido em crescimento, porém não foi possível encontrar associações entre o status do linfonodo e a recorrência do tumor com a expressa marcação. 


\section{Artigo de revisão}

O gene codifica uma proteína de 185 kDa de localização transmembrana e dependente da atividade da tirosina quinase responsável pela emissão da sinalização de crescimento celular. Em consequência, tumores mamários que expressam HER2 são mais agressivos e apresentam uma sobrevida livre da doença e global com taxas inferiores aos que não expressam. ${ }^{27,28}$

Acredita-se que cerca de 15-20\% dos tumores mamários são HER2 positivo e respondem ao anticorpo Trastuzumabe ${ }^{\circledR}$ como primeira linha de tratamento.Em 2005, a liberação do Trastuzumabe ${ }^{\circledR}$ foi uma evolução e esperança no tratamento dos cânceres HER2 positivo, pois age sob a porção extracelular da proteína e inibe a progressão do tumor. ${ }^{29}$

Apesar das ferramentas para o diagnóstico e tratamento de tumores HER2, ainda são constantes as mudanças na clínica para determinar com segurança qual tumor seria propriamente positivo. A heterogeneidade do tumor traz discordâncias quanto à sua marcação e nem sempre a primeira linha do tratamento promove a sensibilidade dos receptores hormonais. Em 2007, Wolffe e colaboradores ${ }^{30}$ da American Society of Clinical Oncology (ASCO) e do CAP definiram as recomendações para a avaliação da proteína HER2 em câncer de mama.

\section{Classificação molecular do câncer de mama}

Noinícioda década passada,comodesenvolvimento das técnicas genômicas, os tumores mamários foram classificados molecularmente. Cada grupo, em acordo com a positividade dos receptores e avaliados por microarranjos gênicos, revelou quatro subgrupos: luminal (A e B), basal-like, superexpressão de HER2 e normal; e, posteriormente, outros dois: claudin-low e mesenquimal. . $^{31,32}$

Os tumores luminais apresentam ER positivo, padrões de expressão gênica das células epiteliais luminais mamárias, incluindo a expressão das citoqueratinas tipo 8 e 18, além de possuírem fenótipo de baixa proliferação celular, expressão elevada de genes relacionados ao ER e bom prognóstico. ${ }^{32}$ Já os tumores luminais tipo B apresentam altas taxas de proliferação, tipo histológico agressivo e prognóstico pior que os luminais A. ${ }^{32}$

O subtipo basal, também conhecido como 'triplo negativo', apresenta RE, RP e HER2 negativos. Apresentam comportamento agressivo, de pior prognóstico e alvo terapêutico não definido. ${ }^{33}$ Constituem cerca de $10-20 \%$ de todos os tumores de mama, afetando mais frequentemente mulheres jovens. Não respondem a tratamentos com drogas antiestrogênicas e monoclonais, além de apresentarem fenótipo quimiorresistente. Novos dados têm enfatizado que a complexidade molecular do câncer de mama triplo negativo constitui muito mais do que um modelo basal. A análise transcriptoma de tumores triplo negativos identificaram seis subtipos, sendo dois tipos basais, um imunomodulatório, um mesenquimal, um mesenquimal do tipo células-tronco e um subtipo luminal receptor de andrógeno. ${ }^{34}$

O subtipo superexpressão do HER2, cujo fenótipo é RE negativo e HER2 positivo, apresenta amplificação do oncogene HER2 e, concomitantemente, superexpressão da proteína. ${ }^{32}$ Estes tumores respondem a drogas que bloqueiam a atividade de HER2, por exemplo, o anticorpo monoclonal Trastuzumabe ${ }^{\circledR}$.

O subtipo normal foi caracterizado por meio do aumento da expressão de muitos genes conhecidos por serem expressos pelo tecido adiposo e por outros tipos de células epiteliais. ${ }^{35}$ Este subtipo, assim como os subtipos claudin-lowe mesenquimal, estão pobremente caracterizados e com significância clínica limitada.

\section{Ferramentas de prognóstico molecular}

O desenvolvimento de técnicas genômicas tem proporcionado a elucidação de mecanismos envolvidos na carcinogênese, em destaque, para genes da regulação e diferenciação celular. Entre as principais alterações da carcinogênese podem ser citadas: a expressão aumentada de oncogenes, inibição de supressores tumorais, instabilidades cromossômicas, alterações epigenéticas, alterações nas vias de reparo, entre outras. Neste cenário, os testes moleculares para o câncer de mama foram desenvolvidos e validados baseados nestas modificações e atualmente exercem papéis funcionais na caracterização do tumor, fornecendo alternativas terapêuticas mais adequadas.

Entre os testes validados pelo Food and Drugs Administration (FDA) - órgão institucional norteamericano responsável pela liberação e fiscalização, equivalente à Agência Nacional de Vigilância Sanitária (ANVISA) no Brasil -, somente o Oncotype DX ${ }^{\circledR}$ e o MammaPrint estão liberados para a rotina clínica. Os demais são complementares e realizados por decisões entre paciente e médico e também quando os tumores não entram nas especificações requeridas do teste. Em geral, são válidos para tumores pequenos, sem metástase, RE positivo e HER2 negativo. 


\section{Oncotype $\mathbf{D X}^{\circledR}$}

O ensaio Oncotype DX ${ }^{\circledR}$ (Genomic Health, Redwood City, CA, USA) avalia a expressão de 21 genes no tecido mamário, seja ele parafinado ou fresco, pela reação em cadeia da polimerase por transcrição reversa em tempo real. O teste consiste na avaliação de 16 genes relacionados ao câncer de mama e cinco genes de referência (GAPDH, ACTB, RPLPO, GUS e TFRC), utilizados como padrões. Os 16 genes incluem aqueles relacionados ao RE (ER, PR, BCL2 e SCUBE2), proliferação (Ki67, STK15, Survivin, CCNB1 e MYBL2), HER2 (HER2 e GRB7), invasão (MMP11 e CTSL2), além de três genes adicionais relacionados (GSTM1, CD68 e BAG1). O resultado da expressão é dado em score de recorrência, que varia entre taxas de zero a 100 para cada paciente. Este score é caracterizado em baixo risco (score $<18)$, intermediário (score entre 18 e 30) e avançado (score $\geq$ $31){ }^{36}$ O Oncotype $D X^{\circledast}$, sem dúvidas, é o mais utilizado na clínica, com maior número de pesquisas de validação e respostas sobre níveis de recorrência e benefícios na ausência de quimioterapia.

Atualmente, existem diversos ensaios clínicos em andamento com o Oncotype $\mathrm{DX}^{\circledast}$ que têm por alvo tumores que não se classificam dentre as validações do teste, mas que poderão beneficiar diversos pacientes.

\section{MammaPrint}

O MammaPrint (Agilent, Amsterdam, The Netherlands) é um teste que se aplica à tecnologia dos microarranjos gênicos. Avalia a expressão de 70 genes, dentre os quais estão: genes do ciclo celular, proliferação, invasão, metástase, angiogênese e transdução de sinal.Diferentemente do Oncotype $\mathrm{DX}{ }^{\circledast}$, o teste fornece resultados em dois perfis: alto risco (taxas elevadas para chances de metástase) e baixo risco (menores taxas para metástase). Os valores para sua validação foram ajustados e aplicados para pacientes que tiveram metástase após cinco anos, linfonodo sentinela negativo e que não receberam tratamento sistemático. ${ }^{37}$

Em suma, o teste é aconselhável para pacientes com idade menor que 61 anos de idade, tumores em estádio I e II, RE positivo ou negativo, linfonodo negativo, exceto para casos em que há acometimento de no máximo três linfonodos. Uma das peculiaridades observadas é que somente no ano de 2013 o teste foi validado para tumores conservados em parafina ${ }^{38} \mathrm{e}, \mathrm{em}$ especial, tumores RE negativos quase em sua totalidade são classificados no score de alto risco. ${ }^{37}$

\section{PAM 50 - Prosigna}

OProsigna (Nanostring Technologies, Inc., Seattle) é um dos testes genômicos mais recentes. Consiste na avaliação de 50 genes, dos quais, oito são normalizadores, seis controles positivos e oito controles negativos da reação. ORNA é isolado do tecido fresco ou em parafina, e consiste na mensuração por hibridização específica das regiões de cada um dos 50 genes, dando como resultado o score de recorrência. Denominado de risk of recurrence score (ROR), os valores são fornecidos entre zero e 100, em três divisões: 1 ) modelo por coeficiente de COX, que inclui teste estatístico de Pearson; 2) score de proliferação; e 3) tamanho do tumor representando agressividade histopatológica. ${ }^{39}$

As categorias de risco são divididas em três (baixa, intermediária e alta), determinando o risco de recorrência após dez anos de diagnóstico. Validado como preciso e sensível, o teste foi aprovado pelo FDA somente como ferramenta-teste de prognóstico, não podendo ser utilizado como determinante para escolha ou mesmo preditivo de acompanhamento de terapia. O material a ser analisado deve ser o RNA isolado através do sistema de análise nCounter ${ }^{\circledR}$ Dx (Nanostring Technologies, Inc., Seattle), eé garantido que apenas $250 \mathrm{ng}$ de RNA são suficientes para todas as análises requeridas do teste. ${ }^{39}$

\section{Limitações e perspectivas dos testes}

Assim como os biomarcadores tradicionais, que apresentam divergências na sensibilidade e especificidade para determinada patologia, os testes gênicos possuem controvérsias em suas validações e nem sempre são ideais para serem aplicados em decorrência da heterogeneidade dos tumores de mama. Por exemplo, em nenhum dos testes aqui exemplificados há um consenso para tumores HER positivos e avançados.

O Oncotype $\mathrm{DX}^{\circledR}$, talvez o mais realizado e confiável entre as validações, apresenta uma faixa de resultado intermediária, que traz dúvidas, para médico e paciente, sobre qual conduta tomar. Quimioterapia ou não quimioterapia? No mesmo cenário, o MammaPrint, embora exclua esta faixa intermediária, apresenta taxas de recorrência superiores ao Oncotype $\mathrm{DX}^{\circledR}$. Por fim, o Prosigna, bastante pretensioso, ainda apresenta dúvidas e ausência de vantagens sobre os demais testes no mercado, sendo necessários mais estudos para comprovar sua eficiência.

Em recente publicação, há crescentes evidências da utilização destes testes moleculares na prática clínica, 


\section{Artigo de revisão}

trazendo validação prognóstica para alto e baixo risco de necessidade de tratamento e quimioterapia, assim como de validação de recorrências a partir de cinco anos da data da cirurgia. ${ }^{40}$

Sem dúvidas, os testes gênicos têm revolucionado o tratamento do câncer no que diz respeito à eliminação de tratamentos agressivos e o custo-efetividade das quimioterapias. Com isso, tem garantido maior segurança para o oncologista na prescrição do tratamento, determinando com individualidade a quimioterapia.

\section{Considerações finais}

Muitos testes que exploram as assinaturas gênicas têm nos permitido responder parcialmente a perguntas sobre prognóstico tumoral, recidivas e outras variáveis do fenótipo tumoral. Com eles, a quimioterapia tem se tornado mais individualizada, precisa e, quando comprovada a sua não necessidade, extinguido situações desconfortáveis para os pacientes, além do melhor custo e economia para o sistema de saúde. Atualmente, diversas validações estão em andamento. Consequentemente, testes reformulados, mais específicos e sensíveis, poderão ser aplicados na rotina clínica em breve.

\section{Referências}

1. Siegel R, Naishadham D, Jemal A. Cancer statistics, 2013. CA Cancer J Clin. 2013;63:11-30.

2. MS - Ministério da Saúde. Instituto Nacional de Câncer (INCA). Disponível em: http://www2.inca.gov.br/wps/wcm/connect/ tiposdecancer/site/home/mama/cancer_mama+. Acesso em: 14 de setembro de 2014, às 23h23.

3. Schnitt SJ, Collins LC. Pathology of benign breast disorders. In: Harris JR, Lippman ME, Morrow M, Osborne CK. Diseases of the breast. $4^{\circ}$ edição. Philadelphia: Wolkers Kluwer; 2010. 69-85.

4. Lakhani SR, Ellis IO, Schnitt SJ, et al. WHO Classification of tumours of the breast. 4th ed. Lyon: IARC Press; 2012.

5. Elston CW, Ellis IO. Pathological prognostic factors in breast cancer. 1. The value of histological grade in breast cancer: experience from a large study with long-term follow-up. Histopathology 1991;19:403-10.

6. Liedke PE, Finkelstein DM, Szymonifka J, et al. Outcomes of breast cancer in Brazil related to health care coverage: a retrospective cohort study. Cancer Epidemiol Biomarkers Prev. 2014;23:126-33.

7. Beam CA, Conant EF, Sickles EA. Association of volume and volume-independent factors with accuracy in screening mammogram interpretation. J Natl Cancer Inst. 2003;19:282-90.

8. Heywang-Köbrunner SH, Schreer I, Heindel W, et al. Imaging studies for the early detection of breast cancer. Dtsch Arztebl Int. 2008;105:541-7.

9. Barlow WE. Performance of diagnostic mammography for women with signs or symptoms of breast cancer. Journal of the Nacional Cancer Institute. 2002;27:2600-08.

10. Helvie MA. Imagin analysis: mammography. In: Harris JR,
Lippman ME, Morrow M, Osborne CK. Diseases of the breast. $4^{\circ}$ edição. Philadelphia: Wolkers Kluwer; 2010. 116-130.

11. Tejerina BA, Tejerina BA, Rabadán DF, et al. Breast imaging: how we manage diagnostic technology at a multidisciplinary breast center. J Oncol. 2012;213-421.

12. Khouri NF. Breast Ultrasound. In: Harris JR, Lippman ME, Morrow M, Osborne CK. Diseases of the breast. $4^{\circ}$ edição. Philadelphia: Wolkers Kluwer; 2010. 131-151.

13. Smith RA. The evolving role of MRI in the detection and evaluation of breast cancer. New Engl J Med. 2007;13:1362-64.

14. Salem DS, Kamal RM, Mansour SM, et al. Breast imaging in the young the role of magnetic resonance imaging in breast cancer screening diagnosis and follow up. J Thorac Dis. 2013;5:9-18.

15. Menezes GL, Knuttel FM, Stehouwer BL, et al. Magnetic resonance imaging in breast cancer: a literature review and future perspectives. World J Clin Oncol. 2014; 5:61-70.

16. Schelfout K, Van Goethem M, Kersschot E, et al. Contrast-enhanced MR imaging of breast lesions and effect on treatment. Eur J Surg Oncol. 2004;30:501-07.

17. Czajka-Oraniec I, Simpson ER. Aromatase research and its clinical significance. Polish Journal of Endocrinology 2010;61:126-34.

18. Caldon CE. Estrogen signaling and the DNA damage response in hormone dependent breast camcers. Front Oncol. 2014;14(4):106. eCollection 2014.

19. Folgueira MAAK, Brentani MM. Câncer de mama. In: Ferreira CG, Rocha JC. Oncologia Molecular. $2^{\circ}$ edição. Rio de Janeiro: Atheneu; 2010. 135-144.

20. Dieci MV, Piacentini F, Dominici M, et al. Quantitative expression of estrogen receptor on relapse biopsy for ER-positive breast cancer: Prognosticlmpact. Anticancer Res. 2014;34:3657-62.

21. Dunnwald LK, Rossing MA, Li Cl. Hormone receptor status, tumor characteristics, and prognosis: a prospective cohort of breast cancer patients. Breast Cancer Res. 2007;12:R6.

22. Abdel-Fatah TM, Powe DG, Hodi Z, et al. High frequency of coexistence of columnar cell lesions, lobular neoplasia, and low grade ductal carcinoma in situ with invasive tubular carcinoma and invasive lobular carcinoma. Am J Surg Pathol. 2007;31:417-26.

23. Ottesen GL, Graversen HP, Blichert-Toft M, et al. Carcinoma in situ of the female breast. 10-year follow-up results of a prospective nationwide study. Breast Cancer Res Treat. 2000;62:197-210.

24. Allred DC, Clark GM, Molina R, et al. Overexpression of HER-2/ neu and its relationship with other prognostic factors change during the progression of in situ to invasive breast cancer. Hum Pathol. 1992;23:974-979.

25. Yang-Feng TL, Schechter AL, Weinberg RA, et al. Oncogene from rat neuro/glioblastomas (human gene symbol NGL) is located on the proximal long arm of human chromosome 17 and EGFR is confirmed at 7p13-q11.2.

26. (Abstract) Cytogenet. Cell Genet. 1985;40:784.

27. Van de Vijver MJ, Peterse JL, Mooi WJ, et al. NEU-protein overexpression in breast cancer: association with comedo-type ductal carcinoma in situ and limited prognostic value in stage II breast cancer. New Eng. J. Med. 1988;319:1239-45.

28. Slamon DJ, Clark GM, Wong SG, et al. Human breast cancer: correlation of relapse and survival with amplification of the HER-2/neu oncogene. Science. 1987;235:177-82.

29. Slamon DJ, Godolphin W, Jones L, et al. Studies of the HER-2/ neu proto-oncogene in human breast and ovarian cancer. 
Lucas Delmonico e cols • A biologia do câncer de mama e testes moleculares de prognóstico

Science. 1989;244:707-12.

30. Romond EH, Perez EA, Bryant J, et al. Trastuzumab plus adjuvant chemotherapy for operable HER2-positive breast cancer. N Engl J Med. 2005;353:1673-84.

31. Wolff AC, Hammond ME, Schwartz JN, et al. American Society of Clinical Oncology/College of American Pathologists guideline recommendations for human epidermal growth factor receptor 2 testing in breast cancer. J Clin Oncol. 2007;25(1):11845.

32. Sørlie T, Wang Y, Xiao C, et al. Distinct molecular mechanisms underlying clinically relevant subtypes of breast cancer: gene expression analyses across three different platforms. BMC Genomics. 2006;26(7):127.

33. Sorlie T, Perou CM, Tibshirani R, et al. Gene expression patterns of breast carcinomas distinguish tumor subclasses with clinical implications. Proc Natl Acad Sci USA. 2001;98:1086974.

34. Mersin H, Yildirim E, Berberoglu U, et al. The prognostic importance of triple negative breast carcinoma. Breast. 2008;17 341-46.

35. Lehmann BD, Bauer JA, Chen X, et al. Identification of human triple-negative breast cancer subtypes and preclinical models for selection of targeted therapies. J Clin Invest.
2006;121:2750-67

36. Peppercorn J, Perou CM, Carey LA. Molecular subtypes in breast cancer evaluation and management: divide and conquer. Cancer Invest. 2008;26:1-10.

37. Paik S, Shak S, Tang G, et al. A multigene assay to predict recurrence of tamoxifen-treated, node-negative breast cancer. N Engl J Med. 2004;351:2817-26.

38. Mook S, Schmidt MK, Viale G, et al. The 70-gene prognosis-signature predicts disease outcome in breast cancer patients with 1-3 positive lymph nodes in an independent validation study. Breast Cancer Res Treat. 2009;116:295-302.

39. Sapino A, Roepman P, Linn SC, et al. Mammaprint molecular diagnostics on formalin-fixed, paraffin-embedded tissue. J Mol Diagn 2013.

40. Parker JS, Mullins M, Cheang MC, et al. Supervised risk predictor of breast cancer based on intrinsic subtypes. J Clin Oncol. 2009;27:1160-7.

41. Coates AS, Winer EP, Goldhirsch A, et al. Tailoring therapies improving the management of early breast cancer: St Gallen International Expert Consensuson the Primary Therapy of Early Breast Cancer 2015. Ann Oncol. 2015 May 4. pii: mdv221. [Epub ahead of print]. 